\title{
Understanding the role of non-covalent interactions in the acridine with different acids of salt molecules
}

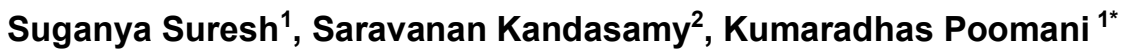 \\ ${ }^{1}$ Laboratory of Biocrystallography and Computational Molecular Biology Department of Physics, \\ PeriyarUniversity, Salem-636 011, India \\ ${ }^{2}$ Faculty of Chemistry, University of Warsaw, Warsaw-02093, Poland
}

convey2sugan@gmail.com

To investigate the salt formation of acridine with 4-amino salicylic acid (I), 5-chloro salicylic acid (II) and hippuric acid (III), the single crystal X-ray structure analysis have been performed. The present study allows to understand the effect of molecular conformation adopted by acridine with hydroxyl group during the stabilization of crystal packing of these salt molecules, and to quantify the propensity of the intermolecular interactions to form the supramolecular assembly. The analysis of atom to atom or residue to residue contacts remains a favoured mode of analyzing the molecular packing in crystals. More importantly, they complement each other and are giving the complete picture of how these molecules assemble in molecular crystals. Hirshfeld surfaces, fingerprint plots and enrichment ratios were generated and further analyzed the intermolecular interactions, and evaluated their quantitative contributions to the crystal packing of the above said three salt molecules (I, II \& III). The non-covalent interaction isosurfaces have employed here, which allows visualizing where the hydrogen bonding and dispersion interactions contribute within the crystal.

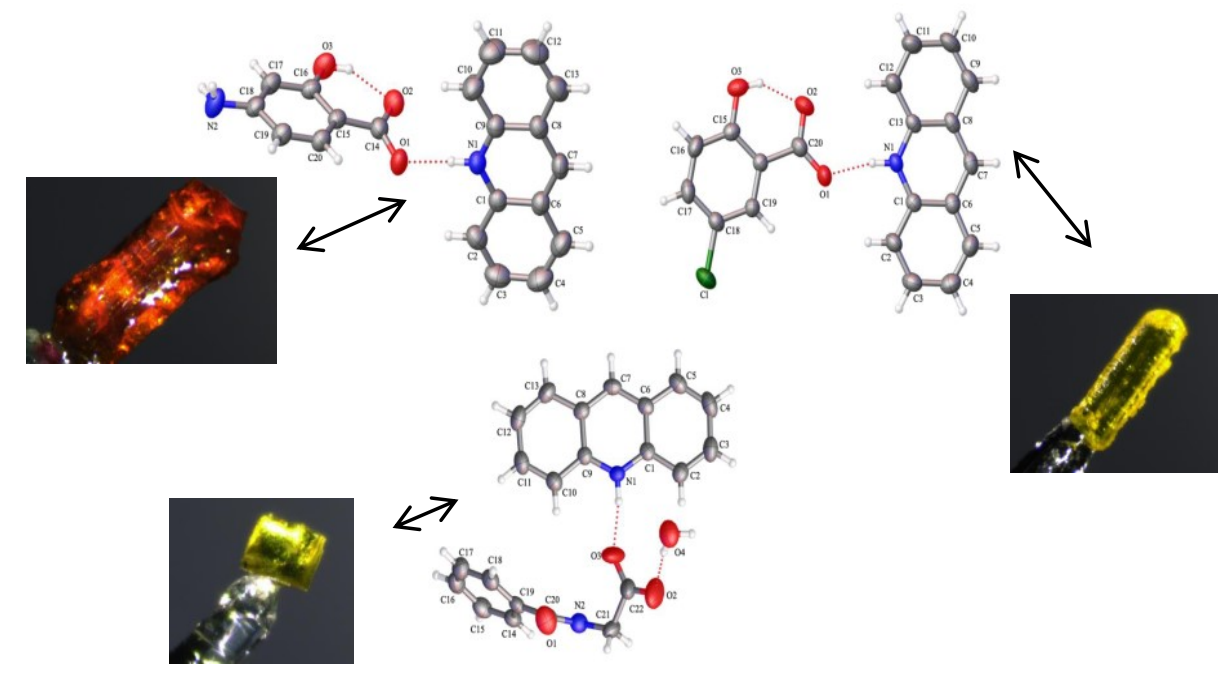

Keywords: Crystal structure; intermolecular interaction; supramolecular assembly; Hirshfeld surfaces; non-covalent interaction 